\title{
Psychopathy domains, sexual experience, and romantic relationship quality
}

\section{Irena Pilch, Klaudia Smolorz}

University of Silesia in Katowice, Poland

irena.pilch@us.edu.pl

\section{INTRODUCTION}

Psychopathic traits have been observed (at a low level) among about $30 \%$ of the population (Coid et al., 2012). Within an evolutionary paradigm, psychopathy can be viewed as personality background for an exploitative and aggressive interpersonal strategy which can be evolutionary adaptive because it predisposes to short-term mating, which can increase a number of lifetime sexual partners. The strategy can incur some important costs to psychopathic individuals (Jonason et al., 2010). These costs can be also observed in the sphere of close relationships.

Sub-clinical psychopathy is usually investigated as one- or twodimensional construct. In this study the triarchic conceptualization of psychopathy was used which proposes three phenotypic constructs: meanness (rebelliousness, callousness, excitement seeking), disinhibition (impulsiveness, negative emotionality, irresponsivity, aggression) and boldness (venturesomeness, emotional resiliency, assertiveness) - a more „positive” aspect of psychopathy (Patrick et al., 2009).

\section{PROBLEM}

1. What are the associations between the triarchic psychopathy dimensions (i.e. boldness, meanness and disinhibition) and indicators of (a) sexual functioning (b) romantic relationship quality? 2. What are the differences between the clusters of participants with similar psychopathy profiles in (a) sexual functioning (b) romantic relationship quality?

\section{METHOD}

Sample. 1291 persons (817 women) from Poland, age $M=24.3$, $S D=5.8$, participated in an on-line survey.

Measures. Psychopathy: a Polish adaptation of the Triarchic Psychopathy Measure (TriPM-41, Pilch et al., 2015; disinhibition $M=0.82$, $S D=0.4, \alpha=.81$, meanness $M=0.87, S D=0.6, \alpha=.84$, boldness $M=1.63$, $S D=0.5, \quad \alpha=.83, \quad$ overall psychopathy $\quad M=1.13, \quad S D=0.3, \quad \alpha=.83)$. Sociosexuality: the Sociosexual Orientation Inventory $(M=3.36, S D=1.6$, $\alpha=.86$; Penke, 2011). One-item measures regarding current romantic relationships with the answers on a 10-point scale (1 "not at all", 10 "completely"): sexual satisfaction ("Are you satisfied with your sexual life?"), relationship satisfaction ("Are you satisfied with your current romantic relationship?”), commitment („How committed are you to maintaining your current relationship?"), feelings ("How intense are your romantic feelings toward your partner?"), costs ("What were the costs that you incurred in your romantic relationship with your partner?") and closeness („How close do you feel to your partner?”).

\section{RESULTS}

The triarchic psychopathy dimensions were associated with sociosexuality and the number of lifetime sexual partners (except meanness). Disinhibition and meanness correlated negatively with sexual satisfaction and the variables characterizing the quality of romantic relationships.

Table 1. Correlations between study variables $(N=1291)$

\begin{tabular}{|c|c|c|c|c|}
\hline & Disinhibition & Meanness & Boldness & Psychopathy \\
\hline Socio-sexuality & $.311^{* *}$ & $.144^{* *}$ & $.198^{* *}$ & $.354^{* *}$ \\
\hline No. sexual partners & $.119^{\star *}$ & .053 & $.106^{* *}$ & $.152^{* *}$ \\
\hline Sexual-satisfaction & $-.072^{*}$ & $-.057^{*}$ & $.196^{*}$ & .051 \\
\hline Relationship-satisfaction & $-.139 * *$ & $-.068^{*}$ & $.098^{* *}$ & -.049 \\
\hline Commitment & $-.169 * *$ & $-.171^{* *}$ & .050 & $-.139^{* *}$ \\
\hline Feelings & $-.121^{* *}$ & $-.144^{* *}$ & .032 & $-.111^{* *}$ \\
\hline Costs & $.153^{*}$ & -.012 & .002 & $.081^{*}$ \\
\hline Closeness & $-.092^{*}$ & $-.101^{* *}$ & $.066^{*}$ & $-.056^{*}$ \\
\hline
\end{tabular}

$* * \mathrm{p}<.001, * \mathrm{p}<.05$.

Two-step cluster analysis was performed on a sub-group of $30 \%$ of participants with the highest psychopathy scores. Four clusters were identified (Silhouette=0.4), which we named „brave-resistant”, „braveimpulsive”, "mean-impulsive” and "mixed”. The ANOVA's with posthoc tests were done to find out the differences between the clusters.

Table 2. Differences between the clusters (ANOVA)

\begin{tabular}{|c|c|c|c|c|c|}
\hline Variable & $\begin{array}{c}\text { Brave- } \\
\text { resistant } \\
N=80\end{array}$ & $\begin{array}{l}\text { Mixed } \\
N=133\end{array}$ & $\begin{array}{c}\text { Brave- } \\
\text { impulsive } \\
N=69\end{array}$ & $\begin{array}{c}\text { Mean- } \\
\text { impulsive } \\
N=99\end{array}$ & $F$ \\
\hline \multicolumn{6}{|c|}{ Triarchic psychopathy dimensions } \\
\hline Disinhibition & $0.7 \mathrm{c} \downarrow$ & $1.0^{b}$ & $1.5^{\mathrm{a}}$ & $1.6^{\mathrm{a} \uparrow}$ & $149 * *$ \\
\hline Meanness & $1.4^{a}$ & $1.4^{a}$ & $0.6^{\mathrm{b}} \downarrow$ & $1.7 \mathrm{c} \uparrow$ & $88^{* *}$ \\
\hline Boldness & $2.5^{\mathrm{a} \uparrow}$ & $1.9 \mathrm{~b}$ & $2.2^{c}$ & $1.6 \mathrm{~d} \downarrow$ & $204^{* *}$ \\
\hline Psychopathy & $1.5^{a}$ & $1.4^{\mathrm{b} \downarrow}$ & $1.5^{\mathrm{a}}$ & $1.6^{\mathrm{a} \uparrow}$ & $18^{* *}$ \\
\hline \multicolumn{6}{|c|}{ Sexuality-related variables } \\
\hline Socio-sexuality & 4.1 & $3.9 \downarrow$ & 4.4 & 4.4 & $2.8^{*}$ \\
\hline No. sex partners & $9.9 \uparrow$ & $4.8 \downarrow$ & 8.7 & 7.6 & 2.4 \\
\hline Satisfaction & $7.4^{\mathrm{a} \uparrow}$ & $6.2^{b}$ & $6.3^{b}$ & $5.9 \mathrm{~b} \downarrow$ & $4.8^{*}$ \\
\hline \multicolumn{6}{|c|}{ Relationship-related variables } \\
\hline Satisfaction & $7.4^{\mathrm{a} \uparrow}$ & $6.7 a b$ & $6.5^{\mathrm{ab}}$ & $5.8^{\mathrm{b} \downarrow}$ & $4.9^{*}$ \\
\hline Commitment & $8.0^{\mathrm{a} \uparrow}$ & $7.3^{a}$ & $7.1 \mathrm{ab}$ & $6.2^{b} \downarrow$ & $6.3^{* *}$ \\
\hline Feelings & $7.5 \uparrow$ & 7.4 & 7.1 & $6.6 \downarrow$ & 2.1 \\
\hline Costs & $4.3^{a b} \downarrow$ & $3.9^{a}$ & $4.8^{a b}$ & $5.0^{\mathrm{b}} \uparrow$ & $3.0^{*}$ \\
\hline Closeness & 4.5 & 4.1 & $5.6 \uparrow$ & $3.9 \downarrow$ & 2.2 \\
\hline
\end{tabular}

Note. Means within a row with the same superscripts do not differ $(p<0.05)$. ${ }^{* *} p<.001,{ }^{*} p<.05$.

\section{CONCLUSIONS}

1. Disinhibition and meanness (but not boldness) were associated with lower quality of romantic relationship.

2. Significant differences between the clusters: brave-resistant (higher boldness, lower disinhibition) and mean-impulsive (lower boldness, higher disinhibition). The latter one showed lower sexual and relational satisfaction and lower commitment and higher perceived costs of the relationship.

3. Boldness was the only psychopathy dimension which was associated with relatively positive outcomes in the sphere of close relationships. 